\title{
Curso clínico e prognóstico do transtorno obsessivo-compulsivo
}

\author{
M ary A M iranda a Isabel A Bordin ${ }^{b}$
}

${ }^{a}$ Setor de Enfermaria do Departamento de Psiquiatria da Universidade Federal de São Paulo/Escola Paulista de Medicina (Unifesp/EPM). São Paulo, SP, Brasil. ' Setor de Psiquiatria Social do Departamento de Psiquiatria e Grupo Inter-Departamental de Epidemiologia Clínica (Gridec) da Unifesp/

EPM. São Paulo, SP, Brasil

\begin{abstract}
The long-term course and prognosis of obsessive-compulsive disorder (OCD) is insufficiently known. To identify predictors of $O C D$ course, follow-up studies of patients with OCD were reviewed and their findings summarized. According to these studies, the following variables were correlated with poor outcome: early onset, severity of OCD symptoms, chronic course, poor social functioning at baseline, lifetime history of comorbid psychiatric and personality disorders, presence of parental Axis I psychiatric diagnosis, and delayed response to serotonin reuptake inhibitors in the beginning of the treatment. Due to the availability of new treatments, most patients with OCD can expect significant improvement, but not complete remission of the disorder. The reviewed followup studies support previous reports on the chronicity of OCD.
\end{abstract}

Keywords Obsessive-compulsive disorder. Comorbidity. Clinical evolution.

\section{Introdução}

O conhecimento do curso, da evolução e do prognóstico de uma doença é fundamental em todas as áreas da medicina. Em psiquiatria, isto é particularmente importante para a validação e a diferenciação das entidades nosológicas. Como a etiologia da maioria das doenças psiquiátricas é desconhecida, o clínico precisa fazer uso de inúmeras variáveis para tentar identificar, diferenciar, tratar e prever a evolução dos transtornos mentais. Assim, estudos que acompanham pacientes com um mesmo grupo de sintomas ao longo do tempo, para determinar a evolução "natural" e o impacto de determinadas abordagens terapêuticas, têm grande importância tanto na diferenciação quanto na validação diagnóstica das doenças mentais. ${ }^{1}$

Apesar de os estudos epidemiológicos apontarem o transtorno obsessivo-compulsivo (TOC) como menos raro do que previamente se supunha, ${ }^{2}$ e de a Organização Mundial da Saúde estabelecer que o TOC é a décima causa de incapacitação, ${ }^{3}$ poucos estudos de seguimento longitudinal com pacientes obsessivos foram desenvolvidos com a finalidade de identificar claramente o curso e o prognóstico desse distúrbio. Os trabalhos existentes apresentam metodologias discrepantes, o que dificulta sua comparação.

Os primeiros estudos sobre a evolução dos estados obsessivos demonstraram que ela é crônica na maioria das vezes, sendo raros os períodos de remissão completa dos sintomas., ${ }^{4,5,7}$ Contudo, esses estudos são retrospectivos e apresentam inúmeros problemas metodológicos: falta de critérios diagnósticos operacionais, dados obtidos por prontuários, não utilização de instrumentos padronizados e indefinição dos conceitos de remissão, recaída, recidiva etc. ${ }^{8}$

Estudos prospectivos mais recentes têm tentado identificar, de forma mais criteriosa, quais fatores interferem no curso e prognóstico do TOC. ${ }^{8-13}$ As variáveis mais estudadas envolvem fatores sociodemográficos (p. ex. idade de início, estado civil) e clínicos (p. ex. características e evolução dos sintomas, resposta terapêutica, comorbidade com outros transtornos mentais e prejuízo sócio-ocupacional). De maneira geral, todos esses estudos concordam que os sintomas obsessivos apresentam flutuações, períodos de melhora e piora, sem que ocorra remissão completa na maioria dos casos. De todo modo, é interessante analisar o valor de cada uma dessas variáveis no adoecer obsessivo.

\section{Início}

Achados da literatura sugerem que o TOC pode iniciar-se em qualquer época da vida. Contudo, há uma concordância entre os trabalhos, mostrando que, na maioria dos casos, o início ocorre na infância ou na adolescência.

O Epidemiologic Catchment Area Study (ECA), estudo epidemiológico americano, entrevistou uma amostra populacional de 20.862 indivíduos e constatou que $64 \%$ dos portadores de TOC apresentaram início dos sintomas antes dos 25 anos de idade, e 74\%, antes dos 30 anos. ${ }^{14}$ Posteriormente, identificaram-se algumas dificuldades no instrumento aplicado para diagnosticar o TOC nesse estudo - o Diagnostic Interview Schedule (DIS) -, colocando em xeque muitos desses achados. Os casos de TOC foram novamente entrevistados, e constatou-se que a idade de início nos casos com diagnóstico estável $(18,7 \pm 13,6$ anos) era inferior àquela previamente observada (25,5 $\pm 16,4$ anos). ${ }^{15}$ Essa revisão epidemiológica só confirmou observações de estudos clínicos de que o TOC instala-se predominantemente na infância, na adolescência e no início da idade adulta.

Para alguns autores, o início precoce estaria associado à 
maior gravidade dos sintomas obsessivos ${ }^{16} \mathrm{e}$ a uma pior evolução. ${ }^{14}$ Campos $^{16}$ observou que naqueles pacientes em que os sintomas começaram mais cedo, a sintomatologia era mais grave (escores mais elevados na Escala de Yale-Brown - YBOCS) e o número de transtornos co-mórbidos era maior que naqueles com início tardio. Skoog \& Skoog, ${ }^{10}$ ao acompanhar uma amostra de pacientes com TOC por 40 anos, comprovaram que o início antes dos 20 anos está associado a uma menor taxa de remissão dos sintomas, sugerindo pior prognóstico. Contudo, a maioria dos estudos que acompanharam pacientes por até 22 anos não conseguiu comprovar a associação entre início precoce e pior evolução., ${ }^{5,811,12,17}$ Essa divergência nos achados pode ser decorrente tanto do reduzido tamanho das amostras na maioria dos estudos de seguimento quanto de variações no intervalo de tempo em que os pacientes foram acompanhados. Dessa forma, como o estudo de Skoog \& Skoog acompanhou os pacientes por longo período, esses autores podem ter sido capazes de identificar o início precoce como uma variável associada à gravidade.

De maneira geral, há uma grande latência entre o início dos sintomas obsessivos e a busca por tratamento - em média, sete anos. ${ }^{1,4,6}$ Essa demora poderia ser parcialmente explicada pelo caráter secreto e reservado dos sintomas, que faz com que esses pacientes evitem ao máximo o contato com psiquiatras e só busquem ajuda quando a intensidade do quadro tenha se acentuado a tal ponto que os familiares e amigos têm de intervir, dispondo-se a acompanhá-los no atendimento médico. ${ }^{18}$

\section{Desencadeantes}

Para Lewis, o fenômeno obsessivo não poderia ser considerado sem que antes se examinasse a própria natureza do homem. ${ }^{7}$ Assim sendo, não é de estranhar que fatores que tenham um forte apelo emocional para o indivíduo estejam relacionados ao desencadeamento dos sintomas. Freqüentemente observa-se que estes estão associados a algum fator precipitante que provocou ansiedade e sensação de impotência, debilidade ou fadiga. ${ }^{6}$ Eventos estressantes antecedendo a instalação do TOC foram identificados em até $70 \%$ dos casos. ${ }^{4-6}$ Fatores desencadeantes relacionam-se geralmente a períodos de maior exigência: rompimentos afetivos, morte de pessoas próximas, puerpério, situações de maior solicitação nos estudos ou no trabalho, mudança de domicílio, troca ou perda de emprego etc. ${ }^{19}$

\section{Curso e duração}

Todos os estudos de seguimento identificam que o TOC tende à cronificação, com baixas taxas de remissão completa. Essa afirmação é tão mais verdadeira quanto maior o tempo de seguimento dos pacientes estudados. De fato, o curso intermitente dos sintomas parece ser mais freqüente no início do transtorno. Com a evolução, a doença tende a se instalar de forma crônica. ${ }^{6,10}$ Em estudo recente, Skoog \& Skoog ${ }^{10}$ demonstraram que, em uma amostra de 144 pacientes, em que a grande maioria nunca havia recebido tratamento, o curso do transtorno diferia na dependência do tempo de seguimento. Quando avaliados no período inicial de dois anos, $56 \%$ dos pacientes apresentavam curso intermitente; $27 \%$, curso crônico; e $17 \%$, episódico. Contudo, após 40 anos de seguimento, a distribuição modificou-se: $31 \%$ apresentaram curso intermitente; $44 \%$, curso crônico; e 10\%, episódico. Mesmo naqueles pacientes com curso crônico, $64 \%$ apresentavam flutuações do quadro psicopatológico, que oscilava entre períodos de exacerbação e redução espontânea da sintomatologia. Esses autores também demonstraram que o curso intermitente estava associado a melhor prognóstico. Nessa casuística, apenas $20 \%$ dos pacientes apresentaram remissão completa dos sintomas, podendo ser considerados "curados". A mesma taxa de cura foi obtida por Steketee, ${ }^{11}$ que acompanhou 100 pacientes por cinco anos.

O curso periódico é observado mais freqüentemente em pacientes do sexo feminino ${ }^{12}$ e, na opinião de alguns autores, relaciona-se a melhor prognóstico. ${ }^{8-10}$ Ainda que o uso de drogas inibidoras da recaptação de serotonina possa reduzir a intensidade dos sintomas obsessivos, não parece interferir no curso da doença ${ }^{8,13} \mathrm{Em}$ um estudo de seguimento com 54 crianças e adolescentes, Leonard et $a{ }^{17}$ observaram que a manutenção de elevados escores de gravidade na quinta semana após o uso de clomipramina estava significativamente associada a uma pior evolução ao longo do tempo. Esse dado parece estar relacionado ao prognóstico, já que outros autores referem achados semelhantes. Segundo Eisen ${ }^{8}$ e Steketee,${ }^{11}$ quanto mais rápido o desaparecimento dos sintomas, maior a chance dessa remissão ser completa e não apenas parcial.

\section{Comorbidade}

Vários autores salientaram que, na fase inicial de instalação do TOC, nem sempre os sintomas têm caráter claramente obsessivo. Sintomas de ansiedade - sobretudo fóbicos ou distúrbios do humor, com manifestações depressivas ou certa disforia acompanhada de tensão e irritabilidade, podem ser observados. ${ }^{1,5,19-21}$

A presença de transtornos co-mórbidos interfere no curso, na evolução e no prognóstico do TOC (vide artigo de Petribú neste volume). Essa questão envolve os seguintes tópicos: (1) os pacientes apresentam um grande número de transtornos comórbidos; (2) há uma freqüente associação do TOC a transtornos do humor, de ansiedade, a transtornos considerados pertencentes ao espectro do TOC (tiques, síndrome de Tourette, compras compulsivas, jogo patológico, compulsão alimentar etc.) e ao abuso e dependência de substâncias; (3) observações relacionadas à seqüência temporal de instalação dos transtornos co-mórbidos revelam que os transtornos de ansiedade (sobretudo fobias) antecedem a instalação do TOC, os transtornos do humor acompanham ou, mais freqüentemente, sucedem o TOC, e os transtornos de abuso e dependência de substâncias sucedem o TOC; $;^{14,19}$ e (4) a intensidade moderada e grave do TOC e a comorbidade psiquiátrica ao longo da vida interferem no funcionamento adaptativo dos pacientes. ${ }^{19}$

De fato, a presença de outros transtornos de ansiedade e do humor, ${ }^{9,11,14,19}$ assim como de transtornos associados ao espectro obsessivo (tiques), ${ }^{17}$ está associada a uma maior gravidade e confere validade diagnóstica ao próprio TOC..${ }^{15}$ Além disso, a 
comorbidade também parece interferir na procura por atendimento médico, que pode relacionar-se a manifestações de ansiedade, depressão, piora dos sintomas obsessivos ou incapacitação profissional. ${ }^{1}$

\section{Evolução}

A maioria dos estudos de seguimento confirma a observação clínica de que o TOC evolui cronicamente e é difícil de ser tratado, com baixos índices de remissão completa dos sintomas: $3,3 \%,{ }^{5} 12 \%,{ }^{8} 16 \%,{ }^{6,4} 20 \%,{ }^{10} 22 \%,{ }^{11} 28 \%{ }^{12}$ e $32 \% .^{7}$

Estudos mais recentes que já examinam o impacto do uso dos inibidores de recaptação de serotonina e da abordagem comportamental constatam que, com os novos tratamentos disponíveis, muitos pacientes podem esperar melhoras significativas ao longo do tempo, mas não remissão completa. Leonard et $\mathrm{al}^{17}$ estudaram 54 crianças e adolescentes com TOC (idade entre 10 e 24 anos), submetidos a várias abordagens terapêuticas, acompanhando-os por um tempo médio de $3,4 \pm 1,0$ anos. Houve remissão completa dos sintomas em apenas três casos $(6 \%)$.
Os estudos de seguimento mais recentes já não discriminam taxas de remissão completa, preferindo usar critérios mais abrangentes que incluam redução nos escores da Y-BOCS e índices de funcionamento adaptativo. Zitterl et $\mathrm{al}^{9}$ estudaram 74 pacientes tratados com fluoxetina e terapia comportamental, encontrando taxas de "bom prognóstico com bom ajustamento social" em $27 \%$ dos casos acompanhados por 18 meses. Orloff et al ${ }^{13}$ seguiram 85 obsessivos por um período médio de dois anos e encontraram redução de até $75 \%$ da Y-BOCS em $31 \%$ da amostra.

\section{Fatores de bom prognóstico}

Até o momento, os dados obtidos sugerem que os seguintes fatores poderiam ser considerados de bom prognóstico: idade de início mais tardia, ${ }^{10,14,16}$ menor gravidade dos sintomas obsessivos, ${ }^{4,5,12}$ ausência de transtornos de personalidade prémórbida, ${ }^{4,5}$ boa resposta inicial à intervenção terapêutica, ${ }^{9,17}$ curso episódico, ${ }^{10}$ menor freqüência de transtornos co-mórbidos, ${ }^{11,13,14,17,19}$ ausência de doenças psiquiátricas nos pais ${ }^{17} \mathrm{e}$ bom ajustamento social. ${ }^{11,19}$

\section{Referências}

1. Goodwin DW, Guze SB, Robbins E. Follow-up studies in obsessional neurosis. Arch Gen Psychiatry 1969;20:182-7.

2. Karno M, Golding JM, Sorenson SB, Burnom A. The epidemiology of obsessive-compulsive disorder in five US communities. Arch Gen Psychiatry 1988;45:1094-9.

3. Murray CJL, Lopez AD, editors. The global burden of disease, Vol I: a comprehensive assessment of mortality and disability from diseases, injuries, and risk factors in 1990 and projected to 2020. Cambridge, Mass: Harvard University Press; 1996.

4. Lo WH. A follow-up study of obsessional neurotics in Hong Kong chinese. Brit J Psychiatry 1967;113: 823-32.

5. Kringlen E. Obsessional neurotics: a long term follow-up. Brit $\mathbf{J}$ Psychiatry 1965;111:709-22.

6. Pollitt J. Natural history of obsessional states: a study of 150 cases. Brit Med J 1957;26:194-8.

7. Lewis A. Problems of obsessional illness. Proc Roy Soc Med 1936;29:325-36.

8. Eisen JL, Goodman WK, Keller MB, Warshaw MG, DeMarco LM, Luce DD et al. Patterns of remission and relapse in obsessivecompulsive disorder: a 2-year prospective study. J Clin Psychiatry 1999;60:346-51.

9. Zitterl W, Demal U, Aigner M, Lenz G, Urban C, Zapotoczky HG et al. Naturalistic course of obsessive-compulsive disorder and comorbid depression. Psychopathol 2000;33:75-80.

10. Skoog G, Skoog I. A 40-year follow-up of patients with obsessivecompulsive disorder. Arch Gen Psychiatry 1999;56:121-7.

11. Steketee G, Eisen J, Dyck I. Predictors of course in obsessivecompulsive disorder. Psychiatry Res 1999;89:229-38.

12. Thomsen PH. Obsessive-compulsive disorder in children and adolescents: predictors in childhood for long-term phenomenological course. Acta Psychiatr Scand 1995;92:255-9.
13. Orloff LM, Battle MA, Baer L, Ivanjack L, Pettit AR. Longterm follow-up of 85 patients with obsessive-compulsive disorder. Am J Psychiatry 1994:151:441-2.

14. Karno M, Golding JM. Obsessive compulsive disorder. In: Robins LN, Regier DA, editors. Psychiatric disorders in America: the epidemiologic catchment area study. New York: Free Press; 1991. p.204-19.

15. Nelson E, Rice J. Stability of diagnosis of obsessive-compulsive disorder in epidemiologic catchment area study. Am J Psychiatry 1997; 154:826-31.

16. Campos MCR. Transtorno obsessivo-compulsivo de início precoce e de início tardio: características clínicas, psicopatológicas e de comorbidade [dissertação]. São Paulo (SP): Universidade de São Paulo; 1998.

17. Leonard HL, Swedo SE, Lenane MC, Rettew DC, Hamburger $\mathrm{SD}$, Bartko JJ et al. A 2 to 7 -year follow-up study of 54 obsessive-compulsive children and adolescents. Arch Gen Psychiatry 1993;50: 429-39.

18. Rasmussen AS, Tsuang MT. The epidemiology of obsessive compulsive disorder. J Clin Psychiatry 1984;45:450-7.

19. Miranda MA. Transtorno obsessivo-compulsivo e comorbidade: um estudo caso-controle [dissertação]. São Paulo (SP): Universidade Federal de São Paulo/Escola Paulista de Medicina; 1999.

20. Esquirol JED. Memórias sobre la loucura y sus variedades. Madrid: Ediciones Dorsa; 1991. p. 378.

21. Janet P. Les obsessiones et la psychasténie. Paris: Félix Alcan Éditeur; 1908. p. 782.

Correspondência: Mary Alves Miranda

Rua Pedroso Alvarenga, 1.046/1.062, conj. 107 - 04531-004 São Paulo, SP

Tel.: (0xx11)3071-2695-E-mail: marymiranda@zaz.com.br 